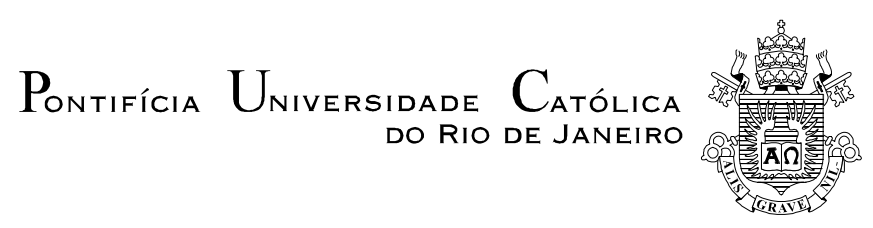

Edinalda Maria de Souza

\title{
Um Estudo sobre um Algoritmo para Visualização de Terrenos
}

Dissertação de Mestrado

Dissertação apresentada como requisito parcial para obtenção do título de Mestre pelo Programa de PósGraduação em Informática da PUC-Rio.

Orientador: Prof. Marcelo Gattass

Rio de Janeiro, agosto de 2003 


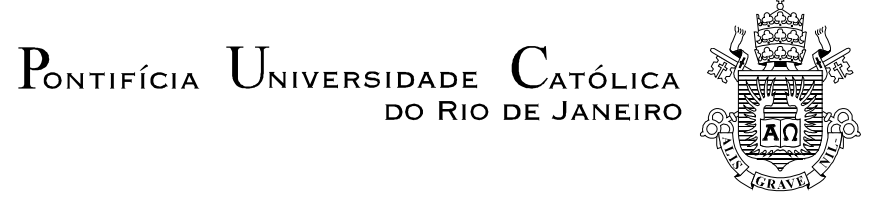

Edinalda Maria de Souza

\section{Um Estudo sobre um Algoritmo para Visualização de}

Terrenos

Dissertação apresentada como requisito parcial para obtenção do título de Mestre pelo Programa de PósGraduação em Informática da PUC-Rio. Aprovada pela Comissão Examinadora abaixo assinada.

\section{Marcelo Gattass \\ Orientador \\ Departamento de Informática-PUC-Rio \\ Prof. Waldemar Celes Filho Departamento de Informática-PUC-Rio.}

Prof. Luiz Fernando Campos Ramos Martha Departamento de Engenharia Civil-PUC-Rio.

Prof. Alberto Barbosa Raposo Departamento de Informática-PUC-Rio.

Prof. Roberto Beauclair Seixas Instituto Nacional de Matemática Pura e Aplicada(IMPA).

Prof. Ney Augusto Dumont Coordenador(a) Setorial do Centro Técnico Científico - PUC-Rio 
Todos os direitos reservados. É proibida a reprodução total ou parcial do trabalho sem autorização da universidade, da autora e do orientador.

\section{Edinalda Maria de Souza}

Graduou-se em Ciências da Computação na UFG (Universidade Federal de Goiás) em 1997. Especializou-se em Redes de Computadores também na Universidade Federal de Goiás em 1999. Trabalhou como Analista de Sistemas na Telegoiás S/A entre 1997 e 2000, onde atuou no desenvolvimento e manutenção de sistemas para transferência de assinaturas telefônicas. Desde 2002 está trabalhando no desenvolvimento das interfaces e algoritmos para visualização dos dados do projeto SISMOSEG3D (parceria Tecgraf/PUC-Rio e Petrobras) cujo principal objetivo é auxiliar na interpretação de dados sísmicos 3D selecionados em camadas.

Ficha Catalográfica



Um estudo sobre um algoritmo para visualização de terrenos / Edinalda Maria de Souza; orientador: Marcelo Gattass. - Rio de Janeiro : PUC, Departamento de Informática, 2003.

[12], 78 f. : il. ; $30 \mathrm{~cm}$

Dissertação (mestrado) ${ }^{-}$Pontifícia
Universidade Católica do Rio de
Departamento de Informática.

Inclui referências bibliográficas.

1. Informática - Teses. 2. Refinamento dependente da visão. 3. Visualização de terrenos. 4. Malha de triângulos. 5. Tempo real. I. Gattass, Marcelo. II. Pontifícia Universidade Católica do Rio de Janeiro. Departamento de Informática. III. Título. 
Aos meus pais, meu irmão e minha irmã. 


\section{Agradecimentos}

Ao meu orientador Marcelo Gattass pelo apoio, dedicação e paciência que tornaram este trabalho possível.

À CAPES e à PUC-Rio pelo apoio financeiro.

À Paula Frederick pela ajuda importante na fase de implementação deste trabalho.

Aos membros da banca examinadora pelas correções e sugestões.

Ao professor Luiz Henrique de Fiqueiredo pelas sugestões.

Ao Centro de Instruções Almirante Sylvio de Camargo da Marinha do Brasil pela utilização do programa SJD-Vis3D e dos terrenos representando a região de Itaóca-ES.

A Anselmo Montenegro pela disponibilização dos terrenos cvzbuffalo e ilha.

À Carolina Alfaro de Carvalho pela revisão do texto desta dissertação.

Aos meus professores de Mestrado Marcus Vinicius Soledade Poggi de Aragão, Paulo Cézar P. Carvalho, Sinésio Pesco, Bruno Feijó e Luiz Velho pelos conhecimentos transmitidos.

Aos colegas e amigos do Tecgraf, do departamento de Informática da PUC-Rio e do Instituto Social do Rio de Janeiro pelas experiências e momentos de descontração compartilhados.

À minha família e a Deus por tudo. 


\section{Resumo}

Souza, Edinalda M.; Gattass, Marcelo(Orientador). Um Estudo sobre um Algoritmo para Visualização de Terrenos. Rio de Janeiro, 2003. 78p. Dissertação de Mestrado - Departamento de Informática, Pontifícia Universidade Católica do Rio de Janeiro.

Algoritmos para visualização interativa de terrenos são complexos e, ao mesmo tempo, de grande importância para muitas aplicações como jogos e planejamento de atividades sobre terrenos. Em função desta complexidade e importância, o tema merecido, na última década, muita atenção da comunidade de pesquisadores em Computação Gráfica e, conseqüentemente, muitas estratégias têm sido desenvolvidas. Entre as mais bem sucedidas estratégias, destacam-se os recentes trabalhos de Lindstrom e Pascucci. O algoritmo proposto por estes autores possui diversas implementações disponíveis na Internet e merece ser reavaliado. Esta dissertação faz esta re-avaliação através de uma implementação independente feita pela autora e testada sobre uma base de terrenos reais. Com o objetivo de tornar esta análise mais completa e dar suporte a algumas conclusões, resultados comparativos de outros algoritmos da área também são apresentados.

\section{Palavras-chave}

Refinamento dependente da visão, visualização de terrenos, malha de triângulos, tempo real. 


\section{Abstract}

Souza, Edinalda M.; Gattass, Marcelo(Advisor). A Study of TerrainVisualization Algorithm. Rio de Janeiro, 2003. 78p. MSc. Dissertation Departamento de Informática, Pontifícia Universidade Católica do Rio de Janeiro.

Algorithms for the interactive visualization of terrains are very complex and, at the same time, of great importance to many applications, such as games and activity-planning over terrains. Due to such complexity and importance, in the past decade this subject has received great attention by researchers on Computer Graphics. As a consequence, a number of strategies have been developed. Among the most successful strategies, one can highlight recent works by Lindstrom and Pascucci. The algorithm proposed by these authors has various implementations available in the Internet and deserves to be reevaluated. The present work makes such reevaluation by means of an independent implementation developed by the author and tested over a base or real terrains. With the purpose of making this analysis more complete and to support some conclusions, comparative results with other algorithms in the area are also presented.

\section{Keywords}

View-dependent refinement, terrain visualization, triangle mesh, real-time. 


\section{Sumário}

1 Introdução

2 Conceitos Básicos e Trabalhos Relacionados 3

2.1. Conceitos Básicos 3

2.1.1. Representação e Reconstrução de Terrenos 3

2.1.2. Métricas de Erro 4

2.1.3. Malha Válida 6

2.1.4. Construção da Hierarquia de Malhas $\quad 7$

2.1.5. Representação por Níveis de Detalhes Dependente da Visão 8

2.1.6. Descarte de Regiões Invisíveis 10

2.1.7. Transições Suaves na Geometria 10

2.2. Trabalhos Relacionados 11

3 Descrição do Algoritmo 15

3.1. Refinamento 17

3.2. Métricas de Erro 19

3.3. Pré-Processamento 20

3.4. Procedimento para Refinamento em Tempo Real 26

3.5. Procedimento para Culling da Malha fora da Visão 30

3.6. Calculando o Frustum de Visão 32

3.7. Procedimento para Geomorphing 37

4 Testes 40

4.1. Breve Descrição da Aplicação 40

4.2. Descrição dos Testes com a Aplicação SJD-Vis3D 40

4.2.1. Verificando o Culling da Malha fora da Visão 46

4.2.2. Verificando o Geomorphing 49

4.2.3. Verificando a Strip de Triângulos 53

4.2.4. Verificando o Pré-Processamento 55

4.2.5. Comparando os Algoritmos de Lindstrom \& Pascucci e de De Boer56 
5 Conclusão

65

5.1. Trabalhos Futuros 


\section{Lista de Figuras}

Figura 1 - Exemplo de Malha produzida pelo algoritmo em estudo.............5

Figura 2 - Erro absoluto e erro relativo. ................................................ 6

Figura 3 - Exemplo de vértice T e aresta sem face. ................................

Figura 4 - Seqüência de malhas da mesma superfície.............................7

Figura 5 - a) Vértice $T$ formado entre diferentes níveis de detalhes; b)

Transição suave entre diferentes níveis de detalhes...........................9

Figura 6 - Modelo de multi-resolução de uma superfície: a) nível 0; b) nível $1 ;$ c) nível 2 e d) nível 3.

Figura 7 - a) Aresta de i para $j$ no DAG; b) Destacando arestas no DAG de

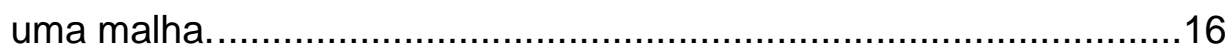

Figura 8 - Malha com esferas envolventes .........................................18

Figura 9 - Terreno dividido recursivamente em blocos...........................21



Figura 11 - Destacando os vértices que aproximam os vértices eventualmente ausentes. .22

Figura 12 - Bloco processado pelo procedimento IniciaErros ....................23

Figura 13 - Os blocos ímpares estão circulados e os pares não. ..............24

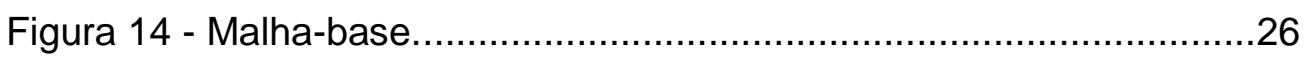

Figura 15 - Destacando filhos direito e esquerdo do vértice j..................27

Figura 16 - Animação da renderização de uma strip de triângulos..........30

Figura 17 - Esferas Envolventes Aninhadas ..........................................32

Figura 18 - Frustum de Visão ..........................................................3

Figura 19 - Caminho sobre o terreno Itaoca1 …...................................42

Figura 20 - Caminho sobre o terreno Itaoca2. ......................................43

Figura 21 - Caminho sobre o terreno Washington 1 ...................................44

Figura 22 - Caminho sobre o terreno Washington2 .................................45

Figura 23 - Comparação do número de triângulos gerados com e sem a

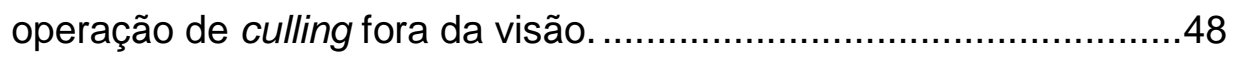

Figura 24 - Quadro do terreno Washington1 …………....................... 49

Figura 25 - Gráficos comparativos entre o número de triângulos desenhados. 
Figura 26 - Gráficos comparativos entre o número total de triângulos ou vértices enviados para o pipeline gráfico e o número de triângulos válidos. .55

Figura 27 - Comparação dos algoritmos de Lindstrom \& Pascucci e de De



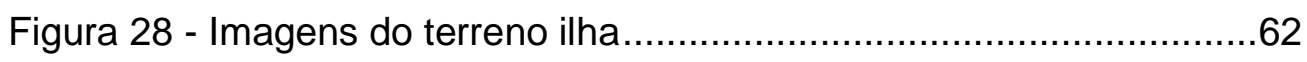

Figura 29 - Imagens do Terreno cvzbuffalo ..........................................63

Figura 30 - Janela do SJD-Vis3D. ................................................

Figura 31 - Diagrama de entradas e saídas das funções TrnPreprocessamento e TrnGeraMalha. ..........................................76 


\section{Lista de Tabelas}

Tabela 1 - Planos que formam o frustum de visão ..................................34

Tabela 2 - Dados dos Terrenos. .........................................................41

Tabela 3 - FPS Médio de cada caminho feito com e sem culling na



Tabela 4 - FPS Médio de cada caminho feito com e sem culling na plataforma 2. ................................................................. 46

Tabela 5 - FPS Médio de cada caminho feito com e sem geomorphing na

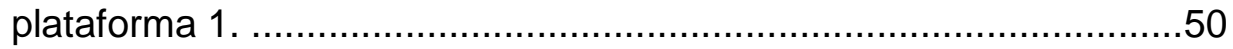

Tabela 6 - FPS Médio de cada caminho feito com e sem geomorphing na

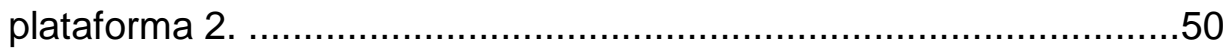

Tabela 7 - Tempo médio do pré-processamento de cada terreno.............56

Tabela 8 - Comparação do caminho Itaoca1 com ambos os algoritmos e

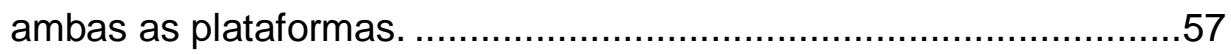

Tabela 9 - Comparação do caminho Itaoca2 com ambos os algoritmos e

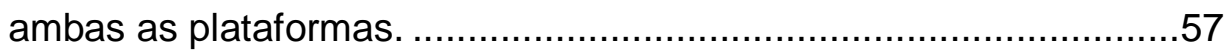

Tabela 10 - Comparação do caminho Washington1 com ambos os

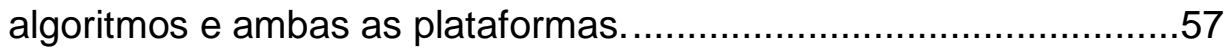

Tabela 11 - Comparação do caminho Washington2 com ambos os



Tabela 12 - Características dos terrenos testados. ..................................61

Tabela 13 - Número de triângulos gerados para o terreno ilha. ...............62

Tabela 14 - Número de triângulos gerados para o terreno cvzbuffalo ......63 